\title{
La capacidad institucional de los consejos de cuenca en México. El caso del Alto Noroeste, 1999-2017
}

\author{
Institutional Capacity of Basin Councils in Mexico. \\ The Case of the High Northwest Basin Council, 1999-2017
}

\author{
Nicolás Pineda Pablos* (D) http://orcid.org/0000-0003-0897-2667 \\ José Luis Moreno Vázquez ** (D) http://orcid.org/0000-0001-8770-434X \\ Rolando Enrique Díaz Caravantes ${ }^{* *}$ (D) http://orcid.org/0000-0002-4117-2197
}

\section{Resumen}

En este artículo se examina la capacidad de los consejos de cuenca de México para gestionar los asuntos hídricos de su territorio, en particular el caso del Consejo de Cuenca del Alto Noroeste. Para ello se revisa literatura académica sobre los componentes de la capacidad institucional, y se proponen variables para evaluar su efectividad a escala local; se analiza el contenido del marco legal de los consejos, y se juzgan sus alcances y limitaciones. Aunque dicho marco es válido para evaluar la política pública en esta materia en México, y también las variables del consejo estudiado, su generalización se deberá verificar y corroborar con otros casos. Se encontró que la Comisión Nacional del Agua controla todos los asuntos del Consejo de Cuenca del Alto Noroeste, y la escasa participación social lograda es inducida y promovida desde arriba. Se concluye que los consejos de cuenca de México carecen de autoridad para llevar a cabo la gestión del agua.

Palabras clave: consejos de cuenca; capacidad institucional; gestión integrada de recursos hídricos; política de aguas; ley de aguas; Sonora.

\section{Abstract}

This article examines the capacity of Mexico's basin councils to manage water issues in its territory, particularly the case of the High Northwest Basin Council. For this purpose, the academic literature on the components of institutional capacity is revised, and variables for evaluating effectiveness at the local level are proposed; the content of councils' legal framework is analyzed, and its scope and limitations are assessed. Although this framework is valid for evaluating public policy on this matter in Mexico, as well as the variables of the council studied, it is necessary to verify its generalization, corroborating it with other cases. It was found that the National Water Commission controls all matters relating to the High Northwest Basin Council, and that the scant social participation achieved is induced and promoted from above. It follows that Mexico's basin councils lack authority to carry out water management.

Keywords: basin councils; institutional capacity; integrated water resources management; water policy; Water Law; Sonora.

Cómo citar: Pineda Pablos, N., Moreno Vázquez, J. L., y Díaz Caravantes, R. E. (2019). La capacidad institucional de los consejos de cuenca en México. El caso del Alto Noroeste, 1999-2017. región y sociedad, 31, e l029. doi: I0.22 I 98/rys20 I9/3 I/I029

* Autor para correspondencia. El Colegio de Sonora, Centro de Estudios en Gobierno y Asuntos Públicos. Obregón \# 54, colonia Centro, C. P. 83000. Hermosillo, Sonora, México. Correo electrónico: npineda@colson.edu.mx

** El Colegio de Sonora, Centro de Estudios del Desarrollo. Obregón \# 54,

colonia Centro, C. P. 83000. Hermosillo, Sonora, México. Correo electrónico: jmoreno@colson.edu.mx

*** El Colegio de Sonora, Centro de Estudios en Salud y Sociedad. Obregón \# 54, colonia Centro, C. P.83000. Hermosillo, Sonora, México. Correo electrónico: rdiaz@colson.edu.mx 


\section{Introducción}

Durante la mayor parte del siglo XX, la política hídrica de México estuvo muy centralizada y concentró las funciones de la gestión del agua en el gobierno federal (Aboites Aguilar, 1998). Incluso la descentralización en la administración pública, realizada a partir de los años ochenta, se ha evaluado como una paradoja, pues está orientada a la centralización política (Rodríguez, 1997). De acuerdo con el artículo 27 constitucional, los recursos hídricos son propiedad de la nación y además se depositan en el gobierno federal, específicamente en el presidente de la república y, de este modo, los gobiernos subnacionales quedan excluidos de los asuntos hídricos. Así, para los gobiernos y los actores locales, los consejos de cuenca, promovidos por la Ley de Aguas Nacionales (LAN), de 1992, (Diario Oficial de la Federación [DOF], 1992) significaron un quiebre en la antigua política hídrica centralizadora, y un paso hacia la descentralización y su involucramiento en el traslado de facultades federales de planeación y gestión de los recursos hídricos. Sin embargo, la creación de dichos consejos, por medio de un acto legislativo central, no significa que se establezcan y funcionen tal y como fueron concebidos por los legisladores y diseñadores de la política. Para que la descentralización y los consejos funcionen y cumplan los fines para los que fueron creados, se requiere que las instancias locales adquieran y tengan una capacidad de gestión de la que han estado privados durante décadas. A más de 25 años de la creación de los consejos de cuenca, el periodo es lo suficientemente largo como para revisar los resultados y alcances de esta reforma.

En este artículo se busca examinar si los consejos de cuenca tienen la capacidad institucional para atender los asuntos hídricos de su territorio, para lo que se plantean estas preguntas: ¿qué tanto se ha descentralizado la gestión del agua hacia los consejos de cuenca?, y iqué capacidad tiene el consejo de cuenca en México y en particular el del Alto Noroeste, para atender los asuntos hídricos del territorio de su jurisdicción?

En el primer apartado se revisa el concepto de capacidad institucional, y se propone una definición operativa que permita explorar su efectividad a escala local. En el segundo se analiza el contenido y los alcances de las disposiciones legales referentes a los consejos de cuenca en México. En el tercero se presenta el caso del Consejo de Cuenca del Alto Noroeste (CCAN) y una revisión de las actas y minutas levantadas en sus sesiones y reuniones de trabajo, para observar su capacidad directiva, operativa y financiera. En el cuarto se discuten los alcances y limitaciones de la capacidad institucional de consejos de cuenca, para terminar con un resumen y una reflexión final a manera de conclusión.

\section{Operacionalización del concepto de capacidad institucional}

El Diccionario esencial de la Real Academia Española (1977) define el término

"capacidad" como la "aptitud, talento, cualidad que dispone a alguien para el 
buen ejercicio de algo" o bien como "el conjunto de condiciones, cualidades o aptitudes, especialmente intelectuales, que permiten el desarrollo de algo, el cumplimiento de una función o el desempeño de un cargo". Se infiere que la capacidad está relacionada estrechamente con las habilidades y la facultad o poder para desarrollar algo; no es un atributo fijo o innato, sino una característica variable que supone un desarrollo o proceso de formación, inducción o educación. Se trata, por lo tanto, de una variable que puede o no estar presente o que puede ser mucha o poca. Su opuesto es la incapacidad, es decir, la carencia de facultad o habilidades para desarrollar una tarea o función.

La capacidad se puede atribuir a un individuo, un grupo de personas, una organización o a una institución. En este caso la institución se define como "las restricciones establecidas por los seres humanos que conforman la interacción humana" (North, 1990, p. 3). Las instituciones se conforman por medio de un entramado de reglas, normas, convenciones y estrategias que guían la actividad de los actores en entornos específicos. Por lo tanto, un marco o arreglo institucional es el conjunto de reglas, procedimientos, usos y costumbres en torno a algún asunto particular. Según el tipo de reglas y usos que prevalezcan, dicho marco puede ser capacitante o discapacitante para determinados actores. Si las reglas y los usos prevalecientes favorecen el buen desempeño de los actores y que, a través de éstos, se alcancen buenos resultados, se puede decir que existe capacidad institucional (Cap-Net United Nations Development Programme [UNDP], 2008).

Por otra parte, aquí la descentralización se entiende como el proceso de trasferir facultades o responsabilidades de una instancia central, que en este caso es el gobierno federal, a otras periféricas ya sea entidades gubernamentales, como los gobiernos de los estados, o bien organizaciones sociales, como los consejos de cuenca (Rodríguez, 1997). La descentralización supone la existencia o la construcción de capacidad institucional en las instancias receptoras de la responsabilidad. Aquí se analiza si los consejos de cuenca tienen la capacidad institucional para hacerse cargo de la gestión del agua de su territorio.

La idea de que los actores locales deben de tener capacidad para planear y administrar los recursos hídricos de su cuenca es una propuesta del modelo denominado "gestión (o manejo) integral de los recursos hídricos" (GIRH), definida como la "toma de decisiones y un manejo de recursos hídricos que toma en cuenta las necesidades y deseos de diferentes usuarios y partes interesadas y se centra en los intereses relativos al uso, control y/o preservación de los sistemas hídricos y su sostenibilidad" (VanHofwegen y Jaspers, 2000, p. 1). Este modelo implica cambiar el anterior de gestión del agua por sectores (agrícola, urbano e industrial), que no se coordinan entre sí, por otro que incluye todos los usos de manera integrada y, sobre todo, que se lleve a cabo en el ámbito local. Para ello, se considera que la delimitación territorial más adecuada para la gestión integrada es la cuenca. Cuando aquí se habla de capacidad institucional de gestión del agua se refiere a que los actores locales organizados tengan las facultades y habilidades para planear y administrar, de manera efectiva, los recursos hídricos de su propia cuenca. 
Para observar y analizar la capacidad institucional en un caso particular se requiere identificar sus componentes e indicadores. Por ello, de manera paralela al enfoque de la gestión integrada del agua, se han desarrollado metodologías, para medir la capacidad institucional de las organizaciones de cuenca. Hooper $(2006,2010)$ propone las dimensiones siguientes: la coordinación en la toma de decisiones; la responsividad de las decisiones; el cumplimiento de objetivos; la sostenibilidad financiera; el régimen de ley; el entrenamiento y construcción de capacidades; la información e investigación; la rendición de cuentas y el seguimiento. Para cada una, Hooper incluye varios indicadores, que suman 115. Esta propuesta de análisis, si bien sirve de antecedente, está enfocada al marco legal y desarrollo político social de Estados Unidos, y requiere información no disponible para los consejos de cuenca de México. Por lo tanto, se necesita un esquema de análisis menos elaborado y enfocado a la situación de la gestión del agua en el país.

Otra propuesta de medición de la capacidad institucional es la de CapNet, una red que promueve la capacidad de las organizaciones de cuenca para su gestión integrada. Para Cap-Net, la capacidad se entiende como el conocimiento, las habilidades y otras facultades asignadas a los individuos, los procedimientos y las reglas de las organizaciones e instituciones del sector (Cap-Net UNDP, 2008). Y considera que las dimensiones principales de la capacidad institucional de las organizaciones de cuenca son: el marco legal, el nivel de autonomía, la efectividad o cumplimiento de los objetivos, la participación de los involucrados, y el financiamiento de las actividades.

Para adaptar esta propuesta al estudio de los consejos de cuenca en México, se puede integrar el marco legal y el nivel de autonomía, de modo que se convierten en una sola dimensión. Asimismo, como el cumplimiento de objetivos tiene problemas de disponibilidad y confiabilidad de la información, se trasforma en el análisis del nombramiento y composición de los órganos directivos. Entonces, para este artículo, el concepto de capacidad institucional de los consejos de cuenca comprende cuatro dimensiones: la autoridad legal, la integración y dirección del consejo, la participación activa de los miembros y la capacidad financiera y cada una tiene, a su vez, sus variables e indicadores respectivos.

La autoridad legal se refiere a las facultades y autoridad que le otorgan las leyes a los consejos de cuenca, para llevar a cabo la gestión del agua de su jurisdicción territorial. La pregunta es si éstos son los encargados directos de la gestión, o más bien consultivos o complementarios de ella. En las disposiciones y figura legal del consejo de cuenca se determina el nivel de autonomía con que cuenta; si tiene personalidad, patrimonio propio y autonomía formal. Por otra parte, la toma de decisiones para la gestión del agua alude a la cantidad o proporción de acuerdos y asuntos que se atienden relacionados con la planeación, las asignaciones y los usos del agua de la cuenca.

La integración y dirección del consejo se refiere a quienes ocupan y controlan los puestos directivos que, por lo general son los de presidente, secretario técnico y otros puestos ubicados en la toma de decisiones y autoridad. Estos puestos los pueden ocupar usuarios o actores de la misma cuenca, o bien ser 
funcionarios de entidades de gobierno, locales o nacionales; es decir, los ocupantes pueden tener un origen social o gubernamental. En el caso de éstos, pueden ser de los gobiernos locales (estatales o municipales) o bien del gobierno nacional (federal). También es relevante observar cómo se nombra a los ocupantes de los puestos de dirección: por designación superior (desde arriba), o por convocatoria a propuestas de los miembros (desde abajo).

Tabla 1. Operacionalización del concepto de capacidad institucional

\begin{tabular}{|c|c|c|}
\hline Dimensiones & Variables & Indicadores \\
\hline \multirow{2}{*}{$\begin{array}{c}\text { Autoridad } \\
\text { legal }\end{array}$} & $\begin{array}{c}\text { Facultades legales } \\
\text { para gestionar el agua }\end{array}$ & $\begin{array}{c}\text { Existencia de facultades legales } \\
\text { para gestionar el agua }\end{array}$ \\
\cline { 2 - 3 } & $\begin{array}{c}\text { Toma de decisiones } \\
\text { para gestión del agua }\end{array}$ & $\begin{array}{c}\text { Número de asuntos y acuerdos } \\
\text { relacionados con la gestión } \\
\text { del agua de la cuenca }\end{array}$ \\
\hline \multirow{2}{*}{$\begin{array}{c}\text { Integración y } \\
\text { dirección del } \\
\text { consejo }\end{array}$} & Integración y estructura & $\begin{array}{c}\text { Proporción de dirección } \\
\text { gubernamental }\end{array}$ \\
\cline { 2 - 3 } $\begin{array}{c}\text { Participación } \\
\text { activa }\end{array}$ & Frecuencia de reuniones & $\begin{array}{c}\text { Proporción de participación } \\
\text { de usuarios de la cuenca }\end{array}$ \\
\cline { 2 - 3 } & Participación de miembros desto director & $\begin{array}{c}\text { Asistencia de los miembros } \\
\text { a sesiones y actividades }\end{array}$ \\
\hline \multirow{2}{*}{$\begin{array}{c}\text { Capacidad } \\
\text { financiera }\end{array}$} & Presupuesto & $\begin{array}{c}\text { Presupuesto anual/volumen } \\
\text { extracción de agua }\end{array}$ \\
\cline { 2 - 3 } & Ingresos propios & $\begin{array}{c}\text { Proporción de recaudación } \\
\text { de cuotas }\end{array}$ \\
\hline
\end{tabular}

Fuente: elaboración propia, derivada de Cap-Net (Cap-Net UNDP, 2008).

La participación activa se refiere a cómo opera o trabaja el consejo en la práctica. Esto se observa por medio de la frecuencia de reuniones y quienes asisten. Para el análisis de los participantes se considera si representan a instituciones locales o a los usuarios o titulares de derechos de agua de la cuenca. La pregunta es qué autoridades locales intervienen, y si todos los usuarios del agua de la cuenca están representados en el consejo.

En la capacidad financiera se toma en cuenta principalmente el tamaño del presupuesto y el origen de los recursos para el sostenimiento del consejo de cuenca, que puede ser de subsidios gubernamentales o bien de recaudación de cuotas de los miembros. El grado de financiamiento externo o gubernamental habla del nivel de dependencia de los gobiernos, mientras que la proporción de recaudación de cuotas señala el de autonomía financiera y fortaleza interna de la agrupación. 
Al considerar y analizar de manera agregada estas dimensiones e indicadores, se intenta inferir un juicio de valor o evaluación aproximada sobre la capacidad institucional del consejo de cuenca estudiado, y también dilucidar en qué medida esta evaluación es generalizable a los demás de México.

\section{La política pública de los consejos de cuenca en México}

En este artículo, la política pública es la voluntad de los órganos de gobierno, principalmente del ejecutivo y del legislativo, manifestada, pero no de manera exclusiva, a través de las leyes y disposiciones legales, de establecer una pauta de acción o una manera de atender algún asunto o problema. En los países con derecho positivo, como México, la legislación constituye la columna de la política pública; la de los consejos de cuenca se define en la Ley de Aguas Nacionales (DOF, 1992); el Reglamento de la Ley de Aguas Nacionales (DOF, 1994) y la Ley de Aguas Nacionales, reformada en 2004 (DOF, 2004).

Ley de Aguas Nacionales de 1992

La LAN de 1992 creó los consejos de cuenca, y los definió como:

Instancias de coordinación y concertación entre la Comisión [Nacional del Agua], las dependencias y entidades de las instancias federal, estatal o municipal y los representantes de los usuarios de la respectiva cuenca hidrológica, con objeto de formular y ejecutar programas y acciones para la mejor administración de las aguas, el desarrollo de la infraestructura hidráulica y de los servicios respectivos y la preservación de los recursos de la cuenca (DOF, 1994, art. 13).

Esta definición denota una intención descentralizadora hacia instancias locales, sobre todo a la participación de los gobiernos estatales y municipales en la gestión del agua; y convertir un gobierno del agua vertical y jerárquico en una red de actores gubernamentales y sociales que interactúan sobre la gestión del agua con capacidades e intencionalidades diferentes (Aguilar Villanueva, 2013). Asimismo, el objeto de "formular y ejecutar programas y acciones para la administración de las aguas [...]” sugiere que estos consejos serían los encargados de la gestión del agua a escala local.

Sin embargo, la ley dispone que los consejos de cuenca se establezcan por decisión o acuerdo del Consejo Técnico de la Comisión Nacional del Agua (CONAGUA) (DOF, 1992, art. 13, segundo párrafo), por lo que no se conciben como una iniciativa de los actores locales, de abajo hacia arriba, sino que es la decisión cupular de un órgano colegiado nacional vinculado con la CONAGUA, para ser ejecutada por los actores locales. Por otra parte, la LAN también dispone que la Comisión promovería y apoyaría la organización de los usuarios e impulsaría su participación en los ámbitos estatal, regional o de cuenca (DOF, 1992, art. 14). 
Esto significa que la organización de los usuarios y los consejos de cuenca son instancias diferentes; la primera se integra por acuerdo de los derechohabientes locales del agua, mientras que los segundos se crean desde arriba, con la concurrencia de los representantes de los gobiernos federal y estatal y de los usuarios.

En resumen, con estas disposiciones principales, la LAN de 1992 comenzó a impulsar la política de descentralización y creación de los consejos de cuenca; los define, dice quién los establece y cómo es su composición general, pero no entraba en detalles.

\section{Reglamento LAN 1994}

En 1994 se expidió el Reglamento de la LAN, el cual detalla algunos aspectos de las disposiciones de la ley. Por ejemplo, establece que los consejos de cuenca estarán integrados como sigue: a) un presidente, el titular de la Secretaría de Agricultura y Recursos Hidráulicos (SARH) que, cuando ésta desapareció, recayó en el titular de la Secretaría de Medio Ambiente y Recursos Naturales (SEMARNAT); b) un secretario técnico, el director general de la CONAGUA, quien suple las ausencias del presidente; c) cinco representantes de la administración pública federal, los titulares de las Secretarías de Hacienda y Crédito Público (SHCP), de Desarrollo Social (SEDESOL), de Energía, Minas e Industria Paraestatal (SEMIP), de Salud (SSA) y de Agricultura, Ganadería, Desarrollo Rural, Pesca y Alimentación (SAGARPA); d) el titular del Poder Ejecutivo de la entidad federativa que corresponda. Cuando la cuenca comprenda más de una, se invitará a los de las otras dentro de la jurisdicción y e) hasta seis vocales de los usuarios, representantes de los diferentes usos, designados por las organizaciones de usuarios y debidamente acreditados por la CONAGUA.

Es relevante observar cómo, a partir de la integración de sus miembros, se ejerce control gubernamental de los consejos: la presidencia recae en el secretario del sector, es decir, la SARH o la SEMARNAT, esto significa que él sería el presidente de los 26 consejos de cuenca, creados en México, a partir de esta ley. Se puede suponer que el secretario no asistirá a las sesiones de los consejos en las regiones del país, y que tampoco se familiarizará con las situaciones y particularidades de cada uno; sin embargo, él preside formalmente los consejos. Esta disposición sobre la presidencia fue modificada con una reforma (DOF, 1997), que estableció que la presidencia recayera en el director general de la CONAGUA, quien tendría voto de calidad. Asimismo, el secretario técnico sería designado por el director general de dicha Comisión, aunque no tendría voto. A pesar de que se sustrajo al secretario del sector de la presidencia, y se descendió un nivel en la jerarquía federal, sigue siendo muy centralizador que el presidente de todos los consejos de cuenca del país sea el director general de la Comisión y, aunque más relacionado con el tema que el secretario del sector, es difícil que asista a las sesiones y esté familiarizado con sus situaciones.

El reglamento señala también que se puede invitar a otros miembros, según se considere conveniente, entre ellos a los ayuntamientos u organizaciones o 
representantes de la sociedad interesados en participar pero es la CONAGUA la que decide y expide las reglas de esta participación.

De este modo, de acuerdo con el marco legal, un consejo típico estaría formado por siete representantes de altos funcionarios federales, uno o más titulares de los gobiernos estatales (de los cuales no se menciona que sean representantes) y otros seis de los usuarios; sería un mínimo de 14 miembros: ocho funcionarios gubernamentales y, de éstos, siete federales. Hay que señalar que los gobiernos municipales y sus organismos operadores de agua potable solo pueden participar en el consejo en calidad de usuarios del agua, y solo uno como representante del uso público-urbano. Está claro el control y dominio federal de los consejos de cuenca. Esta composición puede variar en caso de que, según lo establece el reglamento, "se invite" a más representantes de la sociedad, pero el control de dicha participación lo tendrá la CONAGUA.

En cuanto a las funciones de los consejos de cuenca, en el artículo 16 del reglamento se mencionan las actividades que se resumen a continuación:

- Conocer y difundir los lineamientos generales de la política hidráulica;

- Organizar foros para la participación de las autoridades estatales y municipales, así como de los usuarios y grupos interesados de la sociedad;

- Promover la integración de comisiones de trabajo, para plantear soluciones y recomendaciones de asuntos específicos;

- Concertar con la Comisión las prioridades de uso, así como los mecanismos para enfrentar situaciones extremas;

- Apoyar las gestiones para la concurrencia de los recursos técnicos, financieros, materiales y tecnológicos;

- Participar en los estudios financieros que lleve a cabo la Comisión;

- Participar o intervenir en los demás casos previstos en la ley.

Aquí ya no aparece tan clara la facultad para llevar a cabo la gestión del agua de la cuenca o territorio correspondiente. Los verbos empleados enfatizan actividades subordinadas a la CONAGUA, pero no definen en sentido estricto ninguna facultad para que el consejo de cuenca pueda decidir algo por sí mismo. De esta manera, a pesar de ser un órgano dominado por funcionarios federales, la ley no le asigna la responsabilidad de la gestión del agua de la cuenca.

LAN 2004

En 2004 se reformó la Ley de Aguas Nacionales y se modificaron sustancialmente las disposiciones relativas a los consejos de cuenca, que la nueva ley los define como:

Órganos colegiados de integración mixta, que serán instancia de coordinación y concertación, apoyo, consulta y asesoría, entre la Conagua, incluyendo el Organismo de Cuenca que corresponda, y las dependencias y entidades de las instancias federal, estatal o municipal, y los represen- 
tantes de los usuarios de agua y de las organizaciones de la sociedad, de la respectiva cuenca hidrológica o región hidrológica (LAN art. 3, fracc. XV) (DOF, 2004).

Esta definición hace énfasis en la concurrencia entre los ámbitos de gobierno y la sociedad civil organizada en el seno de los consejos de cuenca, pero como órgano colegiado de "coordinación, concertación, apoyo, consulta y asesoría". No se trata, por lo tanto, de que éstos sean un ente ejecutivo o un encargado de la gestión, sino más bien cajas de resonancia u órganos consultivos. La autoridad administrativa del agua sigue recayendo en el ejecutivo federal, quien delega esta función en la CONAGUA. En todo caso, la LAN 2004 instruye que se promueva la participación de los usuarios y la descentralización de funciones "dentro del marco legal vigente" (DOF 2004 art. 4 y 5). Hay que apuntar, además, que esta nueva versión de la LAN declara de utilidad pública "la gestión integrada de los recursos hídricos, superficiales y del subsuelo, a partir de las cuencas hidrológicas en el territorio nacional, como prioridad y asunto de seguridad nacional" (art. 7, fracc. 1). Existe una tensión evidente en estas disposiciones que, por un lado, mantienen íntegra la centralización, pero fomentan la descentralización; concentran funciones de gestión, pero promueven la coordinación y la participación; declaran de utilidad pública la gestión integrada del agua, que es un enfoque que promueve la gestión local en la cuenca, pero mantienen todo el poder en la instancia federal centralizada. La LAN de 2004 aclara también que los consejos de cuenca no están subordinados a la CONAGUA ni a los organismos de cuenca (art. 13), sin embargo, ésta los preside y ocupa las posiciones principales dentro de ellos.

La LAN 2004 modifica la estructura interna de la Comisión y crea los organismos de cuenca, que ahora son los encargados de la gestión integrada de los recursos hídricos a escala regional. Estas dependencias nuevas aparentan ser descentralizadas, con jurisdicciones regionales relacionadas con las cuencas hidrológicas; sin embargo, la ley les da una autonomía muy acotada y abarcan muchas cuencas y regiones grandes en varios estados. Los organismos de cuenca se definen como: "unidades técnicas, administrativas y jurídicas especializadas, con carácter autónomo que esta Ley les confiere, adscritas directamente al Titular de la Comisión" (art. 12 bis 1). La tensión es clara entre la autonomía y el hecho de estar adscritos al titular, dos atributos que se contradicen.

Ahora los consejos de cuenca se integran por un presidente, un secretario técnico y vocales, con voz y voto, que representen a la federación, los estados y los municipios, a los usuarios del agua (agrícolas, público-urbanos, industriales, pecuarios, acuícolas y de servicios) y a las organizaciones de la sociedad. Ya no se da mayor preeminencia a los representantes federales, y se busca más equilibrio entre los tres ámbitos de gobierno y los representantes sociales. Las entidades federativas están representadas por los titulares del gobierno estatal, quienes pueden nombrar representantes. Asimismo, la LAN de 2004 establece que los vocales de la sociedad civil, que pueden ser usuarios del agua o representar a organizaciones ciudadanas, deben de ser cuando menos 50\%. Es importante señalar, sin embargo, que esta ley considera a los representantes de 
los organismos de agua potable y saneamiento, que por lo general son entidades de los gobiernos municipales, como usuarios sociales. Asimismo, los de los gobiernos estatales y municipales deben ser 35\% como máximo. De este modo, los representantes del gobierno federal serían alrededor de 15\%. Sin embargo, quien represente a la CONAGUA fungirá siempre como secretario técnico del consejo con voz y voto, y la presidencia recaerá en el director general de la dependencia, al menos en su etapa inicial.

Tabla 2. Integración del consejo de cuenca según la LAN 2004

\begin{tabular}{|c|c|c|}
\hline Sector & Vocales & Representación y proporción \\
\hline \multirow[t]{2}{*}{ Gobierno } & $\begin{array}{l}\text { Gobierno } \\
\text { federal }\end{array}$ & $\begin{array}{c}\text { SEMARNAT, SHCP, SEDESOL, SEMIP, } \\
\text { Secretaría de Economía, SSA y Secretaría } \\
\text { de Agricultura, Ganadería, Desarrollo Rural, } \\
\text { Pesca y Alimentación, aproximadamente 15\% }\end{array}$ \\
\hline & $\begin{array}{l}\text { Gobiernos estatales } \\
\text { y municipales }\end{array}$ & No más de $35 \%$ \\
\hline Sociedad & $\begin{array}{l}\text { Usuarios y } \\
\text { organizaciones } \\
\text { ciudadanas }\end{array}$ & $\begin{array}{l}\text { Incluye a los organismos operadores } \\
\text { de agua potable y saneamiento, al menos 50\% }\end{array}$ \\
\hline
\end{tabular}

Fuente: DOF (2004), con modificaciones.

Las funciones o tareas encomendadas a los consejos de cuenca aumentan en número y temas, pero todas se mantienen en el espacio de la "coordinación, concertación, apoyo, consulta y asesoría”; los verbos predominantes entre las funciones asignadas son: contribuir, participar, promover, coadyuvar, colaborar, impulsar, auxiliar y apoyar. En cambio, no incluye otros como gestionar, decidir, asignar, sancionar o planear. Por lo tanto, en este rubro no hay avance en las facultades de los consejos, ni se les empodera para que realicen funciones de gestión o administración del agua de sus cuencas.

La integración de los consejos, dirigida y controlada por la Comisión Nacional del Agua, así como las funciones subordinadas y complementarias que se le asignan contrastan con las recomendaciones de la LAN 2004 en los artículos 14 y 14 bis del capítulo "Organización y participación de los usuarios y de la sociedad" en donde dice que la Comisión "promoverá y facilitará la participación de la sociedad en la planeación, toma de decisiones, ejecución, evaluación y vigilancia de la política nacional hídrica" (art. 14 bis). Aquí sí se refiere directamente a la participación de los usuarios en la gestión del agua, pero solo como exhortación, sin darles ningún instrumento ni responsabilidad concreta. 
En cuanto a los cambios y énfasis nuevos que esta ley hace en la integración de los consejos de cuenca con respecto al reglamento de 1994, el transitorio segundo de la LAN de 2004 señala que: "En tanto se expiden los reglamentos derivados de la presente Ley, quedan vigentes las disposiciones del Reglamento de la Ley de Aguas Nacionales, en todo lo que no contravenga lo dispuesto en el presente Decreto y la Ley que contiene". Entonces, esta ley actualiza y prevalece sobre los contenidos del reglamento anterior sin derogarlos, sino que más bien los complementa.

\section{El caso del Consejo de Cuenca del Alto Noroeste}

El CCAN fue constituido el 13 de marzo de 1999, en Hermosillo, Sonora, en una reunión a la que asistieron el director general de la CONAGUA y el gobernador del estado, varios presidentes municipales y, como invitado especial, el gobernador de Chihuahua. El punto dos del acta constitutiva señala que este consejo está integrado por un presidente, que es el director general de la Comisión; el titular del Poder Ejecutivo de Sonora; vocales representantes de los usuarios del uso agrícola, público urbano, industrial y pecuario, y el secretario técnico, que es el gerente regional noroeste de la Comisión. Todos asistieron y firmaron el acta (CONAGUA, 1999). Es importante señalar que en ella no aparecen los representantes de otras dependencias federales, ni sectores de la sociedad civil, diferencias relevantes con lo previsto en el marco legal.

El anexo 2 del acta constitutiva del CCAN señala que comprende 100887 $\mathrm{km}^{2}$, y está ubicada en 40 municipios de la esquina noroeste de Sonora. A pesar de que el marco legal señala que la jurisdicción de los consejos se hará con base en cuencas, el acta constitutiva del CCAN no las menciona, y establece la jurisdicción territorial basada en la división política de los municipios (CONAGUA, 1999). El área geográfica del CCAN es desértica, comprende tres cuencas de los ríos Sonoyta, Concepción y Sonora; las corrientes de los dos primeros son efímeras, solo corren en la temporada de lluvias (que son pocas), y permanecen secos la mayor parte del año (Dunbier, 1968, p. 74). En cambio, el río Sonora nace en la sierra, recoge más agua y es un poco más constante y lleva más corriente. Se trata entonces de un área muy extensa donde el agua es un recurso escaso y con una problemática variada.

Después de la creación del CCAN y en un proceso descendente, se establecieron varios organismos auxiliares para la participación social en las cuencas, subcuencas y acuíferos comprendidos en su área territorial. Así en el acta del 19 de septiembre de 2003, se acordó “la integración de los Comités Municipales de Playas Limpias de Puerto Peñasco y Bahía de Kino, como Organismos Auxiliares". En el acta del 10 de noviembre de 2006, "se reconoce como órganos auxiliares a la Comisión de Cuenca del Río Concepción, a la Comisión de Cuenca del Río Sonora, así como a los Comités Técnicos de Aguas Subterráneas de los Acuíferos El Zanjón, Río San Miguel y Mesa del Seri-Victoria” (CONAGUA-Organismo de Cuenca del Noroeste [OCNO], 2017). 
Figura 1. Territorio y división municipal de la jurisdicción del CCAN

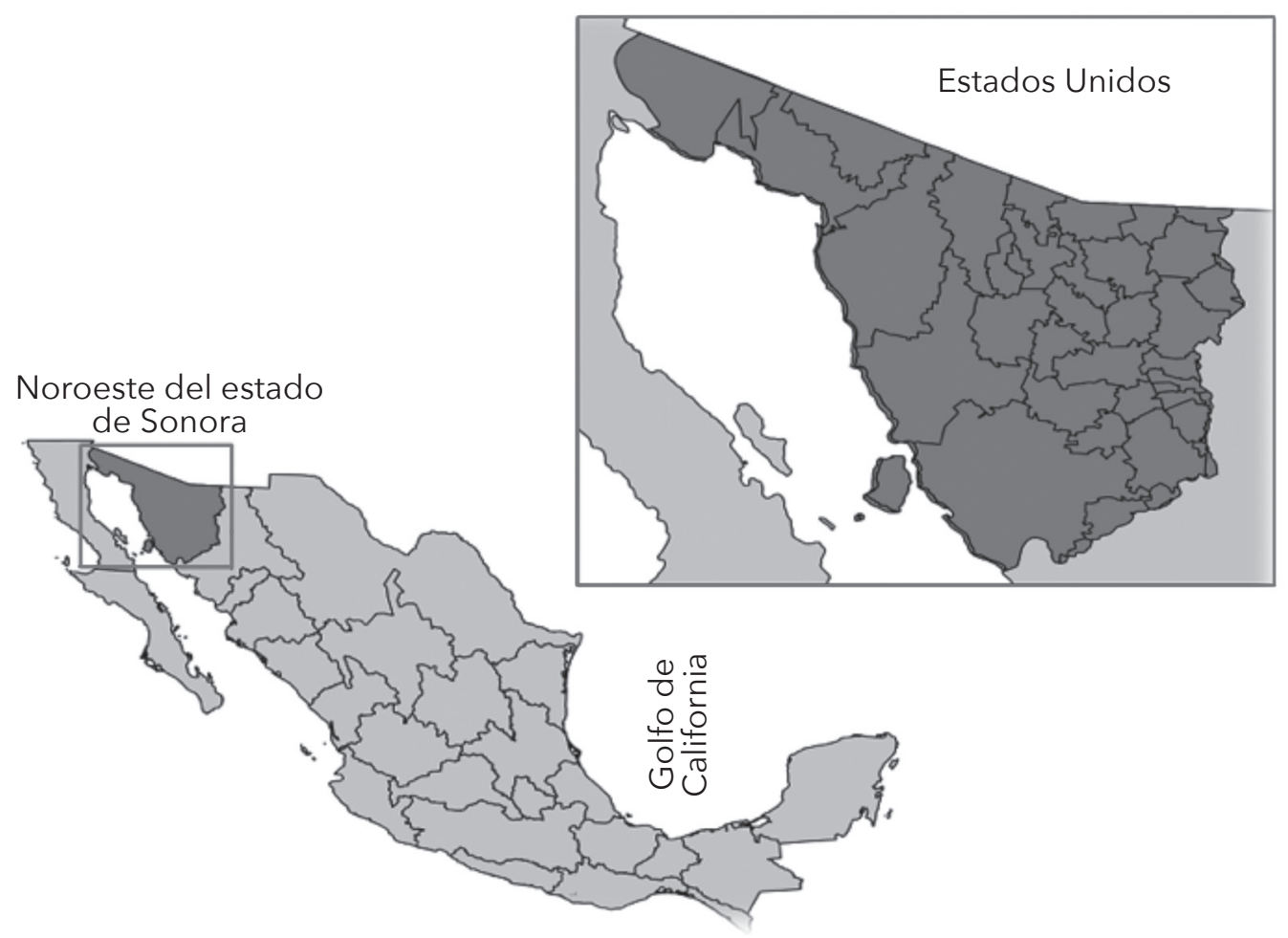

Fuente: elaborado por Alejandro Navarro, con base en datos de la CONAGUA.

Las minutas de las reuniones de trabajo

Este artículo está basado en la revisión y estudio de 73 actas de las sesiones del CCAN proporcionadas por la CONAGUA. Estas actas contienen los datos siguientes: a) una portada o encabezado, por ejemplo: "Minuta de la décima primera reunión de trabajo del Grupo de Seguimiento y Evaluación del Consejo de Cuenca del Alto Noroeste"; b) lugar y fecha: se celebraron en Hermosillo, Sonora, se incluye el día, mes y año, así como el lugar que, por lo general, es en un hotel. Después aparecen estos puntos: I. Antecedentes; II. Intervenciones, donde se mencionan los nombres de los asistentes y sus cargos; III. El motivo de la reunión; IV. El orden del día, con los temas que se abordaron y los exponentes; V. El desarrollo de la reunión, que consiste en una relatoría breve de las participaciones; VI. Acuerdos y cierre del acta. Al final aparecen las firmas de los participantes principales.

Las actas revisadas fueron desde la sesión de instalación del CCAN, 19 de marzo de 1999, hasta la del 23 de noviembre de 2017, con la cual se cerró el periodo de estudio de este artículo; las actas se revisaron y se ordenaron, a fin de evitar duplicados o detectar faltantes. 
De los 73 documentos estudiados, 61 minutas corresponden a reuniones ordinarias del grupo de seguimiento y evaluación (GSE); los 12 restantes son del acta constitutiva, de una reunión extraordinaria, de dos asambleas de usuarios, de seis reuniones de la comisión de operación y vigilancia (COVI) y de dos "sesiones del consejo". Al indagar sobre las diferencias entre los tres últimos tipos de sesiones, se aclaró que el GSE corresponde a la etapa en que el consejo era presidido por el director general o el gerente del organismo de cuenca de la Conagua, el cual cambió de nombre a COVI, cuando la presidencia pasó a manos de un usuario. La sesión extraordinaria se convocó para recibir "comentarios y observaciones sobre la versión preliminar del Programa Nacional Hidráulico y la propuesta de inversiones 2002 para el Noroeste". Por su parte, las asambleas de usuarios son las reuniones en las que se elige o aprueba a representantes nuevos de los sectores de usuarios.

Número de sesiones y asistencia a las reuniones del consejo

En 19 años, el CCAN tuvo 73 sesiones formales, a partir de su instalación hasta cuando concluyó el periodo estudiado, un promedio anual de 3.84 sesiones. El año con más reuniones fue el 2000 cuando se celebraron nueve reuniones, mientras que en 2003, 2009 y 2010 solo hubo dos por año. En la Figura 2 se aprecia una tendencia a la baja en el número de reuniones anuales, hasta 2014, y un repunte ligero en 2015 y 2016.

Figura 2. Número de reuniones por año

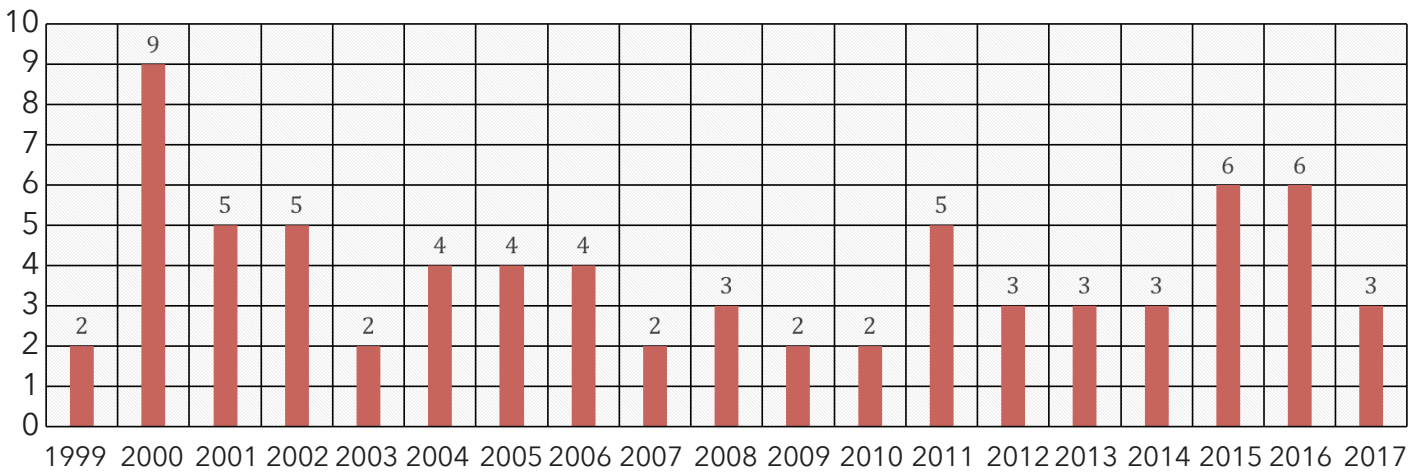

Fuente: elaboración propia, con base en las actas del CCAN.

En cuanto a la asistencia a las sesiones, el promedio de asistentes fue de 20.7. La más concurrida fue la número 68 (28 de septiembre de 2016), con 53 personas, siguió la 35 ( 22 de noviembre de 2006), con 47, según la minuta. En cambio, la sesión 17 (20 de marzo de 2002) tuvo la menor participación, con solo cuatro asistentes. En general, se aprecia una tendencia a la alza en la asistencia a las sesiones del CCAN. 


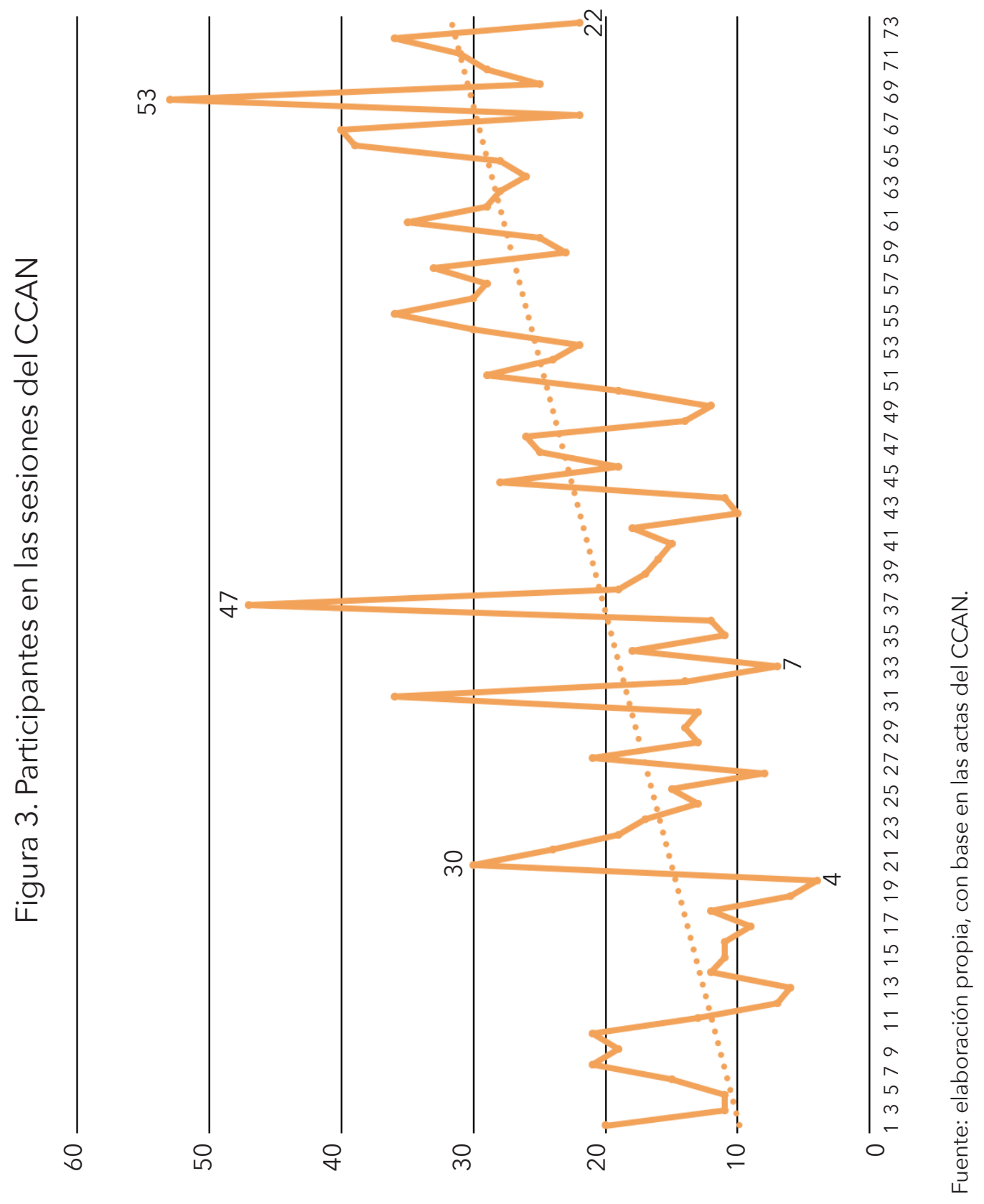


Más allá del número de asistentes, es importante observar de dónde provienen los participantes o las instituciones que representan. La única institución que asistió a las 73 sesiones fue la CONAGUA, que es la organizadora y cuyo representante ha presidido las reuniones (ver Tabla 3). Además, hay una lista larga de organismos e instituciones que han asistido en algunas ocasiones, entre éstos la Universidad Estatal de Sonora y El Colegio de Sonora; presidentes municipales, como el de Caborca, Nogales y Altar; organismos operadores de agua de varios municipios integrantes del CCAN y muchas otras dependencias de diferentes órdenes de gobierno y organizaciones de la sociedad civil, que asistieron a menos de 10 reuniones en los 19 años del periodo de estudio.

Tabla 3. Entidades que asisten a las sesiones del CCAN

\begin{tabular}{|c|c|c|}
\hline Entidad & Asistencias & $\%$ \\
\hline CONAGUA & 73 & 100 \\
\hline $\begin{array}{c}\text { Comisión Estatal del Agua (CEA), antes Comisión } \\
\text { de Agua Potable y Alcantarillado del Estado de Sonora }\end{array}$ & 53 & 73 \\
\hline Vocales representantes de usuarios & 50 & 68 \\
\hline Comités Técnicos de Aguas Subterráneas & 37 & 51 \\
\hline SEMARNAT & 28 & 38 \\
\hline SAGARPA & 27 & 37 \\
\hline SEDESOL & 21 & 29 \\
\hline Universidad de Sonora & 21 & 29 \\
\hline Comisión del Río Concepción & 21 & 29 \\
\hline Comisión Nacional Forestal en Sonora & 21 & 29 \\
\hline Procuraduría Federal de Protección al Ambiente & 15 & 21 \\
\hline Usuarios de los comités locales & 14 & 19 \\
\hline Comisión de Ecología y Desarrollo Sustentable & 12 & 16 \\
\hline
\end{tabular}

Fuente: elaboración propia, con base en las actas del CCAN.

Se observa que, con excepción de los representantes de la CONAGUA, la asistencia de los representantes de las demás instituciones es bastante irregular y variable. La más asidua es la representación del gobierno de Sonora, por medio de las dos comisiones de agua que han existido en el periodo. Asimismo, los vocales gubernamentales federales, como la SAGARPA, la SEMIP, la Secretaría de Economía y la SSA, que ocupan un lugar preeminente en el diseño legal de los consejos de cuenca, tienen poca presencia en las reuniones.

Otro punto relevante que se observa es quién preside las sesiones del CCAN; la de instalación fue presidida por el director general de la CONAGUA, como lo 
prevé la LAN, como presidente de los consejos de cuenca. Sin embargo, después dicho funcionario no volvió a asistir, y su función la asumió personal de la Comisión, como está asentado en las actas. Las sesiones son organizadas y presididas indistintamente por alguno de estos funcionarios: a) gerente regional o director general del CCAN; b) subgerente regional de Programas Rurales y Participación Social o coordinador de Atención a Emergencias y Consejos de Cuenca y c) jefe de Proyectos de Consejos de Cuenca.

Aunque el gerente regional es el suplente formal, para presidir el consejo de cuenca, no asistió a la tercera parte de las sesiones, y fue representado por el subgerente u otro funcionario de la CONAGUA. La sesión del 13 de agosto de 2005 contiene incluso un acuerdo que dice: "solicitar al gerente regional que asista a las sesiones".

Está claro el predominio y la dependencia que el CCAN tiene con respecto a la Conagua y la poca participación de las otras dependencias federales. Además, se observa que las partes más interesadas y que asisten con más frecuencia, aunque con pocas facultades legales, son los representantes del gobierno estatal y de los diferentes sectores de usuarios.

El financiamiento del CCAN

Desde 2008, la CONAGUA y la CEA firman anualmente un convenio para "conjuntar y promover acciones y recursos para incidir en la gestión integrada de los recursos hídricos en el ámbito del Consejo de Cuenca del Alto Noroeste, mediante el fortalecimiento de su gerencia operativa" (CEA, 2013). La cantidad aportada por estas dependencias ha variado, en 2008 fue de $\$ 1300$ 000, que es el monto más elevado que ha recibido. En el resto de los años, recibió entre un millón y $\$ 1260000$. Sin embargo, en 2017 el monto total fue de $\$ 670000$ (CONAGUA-CEA, 2008-2017).

Tabla 4. Financiamiento de la CONAGUA y la CEA al CCAN

\begin{tabular}{|c|c|c|c|}
\hline Año & Aportación CONAGUA & Aportación CEA & Presupuesto CCAN \\
\hline 2008 & 650000 & 650000 & 1300000 \\
\hline 2009 & 500000 & 650000 & 1150000 \\
\hline 2010 & 500000 & 650000 & 1150000 \\
\hline 2011 & 600000 & 600000 & 1200000 \\
\hline 2012 & 400000 & 600000 & 1000000 \\
\hline 2013 & 530000 & 600000 & 1130000 \\
\hline 2014 & 600000 & 600000 & 1200000 \\
\hline 2015 & 630000 & 630000 & 1260000 \\
\hline 2016 & 500000 & 500000 & 1000000 \\
\hline 2017 & 335000 & 335000 & 670000 \\
\hline
\end{tabular}

Fuente: elaboración propia, con datos extraídos de CONAGUA-CEA (2008-2017). 
Por medio de estos convenios se asignan fondos financieros al CCAN, a fin de que sufrague tanto su gasto corriente como la realización de estudios. Así, el firmado el 31 de enero de 2014 le asignó $\$ 1200$ 000, de los cuales $\$ 600000$ los aportó la CONAGUA, provenientes del Ramo 16 del presupuesto federal y otros $\$ 600000$ de la CEA, de recursos propios, destinados a "estudios y proyectos, así como a actividades operativas autorizadas en el seno del Comité Técnico de Seguimiento y Evaluación”.

De acuerdo con el anexo técnico de dicho convenio, la aportación de la Conagua se destina a rubros de gasto corriente: $\$ 300000$ para honorarios, 92000 para pago de arrendamiento, 60000 para servicios de capacitación, y 44000 para traslados y viáticos, gastos menores como combustible, equipamiento y mobiliario, acceso a internet y otros servicios básicos.

Con base en estos convenios, en 2014 se encargó el estudio "Identificación de diques y represos de uso industrial, pecuario y agrícola, para efectos de su uso sustentable e impacto ambiental en el Río Sonora". Asimismo, en 2015, se realizó otro: "Evaluación de la calidad toxicológica del agua suministrada a la ciudad de Hermosillo". En 2016, se encargó el "Proyecto ejecutivo para la construcción de obras de infiltración para la recarga artificial del acuífero Mesa del Seri-La Victoria”. La gerente informó que el CCAN no contaba con fondos de otras fuentes ni se cobraban cuotas a los usuarios (Mercadante, 2017).

Como se puede observar, el CCAN depende enteramente de la CONAGUA y de la CEA para su sostenimiento, y los montos recibidos se pueden considerar precarios y escasos para lograr el objetivo que, según el acta constitutiva es: "Formular y ejecutar programas y acciones para la mejor administración de las aguas nacionales, el desarrollo de la infraestructura hidráulica y de los servicios respectivos y la preservación de los recursos de la cuenca" (CONAGUA, 1999).

\section{Fortalecimiento institucional del CCAN}

Desde su instalación, el CCAN ha ido evolucionando y agregando algunos elementos orientados a fortalecerlo y a aumentar su capacidad institucional, como contar con gerente, es decir, con un empleado permanente encargado de llevar sus asuntos, así como con un local u oficina que sea la sede. De este modo, el grupo de seguimiento y evaluación del CCAN acordó, el 3 de octubre de 2007, la instalación de la gerencia operativa y a partir de ese año el gobierno de Sonora se comprometió a aportar recursos para su operación.

Después de revisar el modelo del reglamento proporcionado por la CONAGUA, en 2010, y de lanzar, en 2012, una convocatoria para hacer comentarios y modificaciones a la propuesta de documento, el 7 de marzo de 2013, el CCAN aprobó sus propias reglas de operación y funcionamiento, de este modo formalizó su organización y procedimientos internos y cuenta con un reglamento interno.

A partir de la aprobación de las reglas de operación y de la disposición que contienen para elegir como presidente a uno de los usuarios, se promovió el "perfeccionamiento" del CCAN, es decir, que su presidente ya no sea el director 
general del organismo de cuenca de la Conagua, sino uno de los usuarios. Así, el 26 de noviembre de 2015 se eligió a un presidente nuevo del CCAN, al ingeniero Luis Alfonso Robles Contreras, quien además era el alcalde de Magdalena, Sonora. Sin embargo, el secretario técnico del CCAN, quien organiza las sesiones y lleva los asuntos del consejo, continúa siendo un funcionario de la Comisión.

De este modo, el CCAN desde 2008 cuenta con un gerente y con los fondos aportados por la CONAGUA para renta de un local, que le sirve de oficina para atender sus asuntos. Asimismo, desde 2013 tiene un reglamento propio y a partir de 2015 fue "perfeccionado", al elegir a un usuario de agua como presidente.

Acuerdos y agenda de trabajo

Por último, una tarea central de este artículo es la revisión de los acuerdos plasmados en las actas de las sesiones del CCAN. Ahí se puede observar el tipo de asuntos que se tratan y dar seguimiento a algunos de ellos. Uno interesante, por ejemplo, es la creación de un centro de información y consulta sobre el agua (CICA) de las cuencas de los ríos Sonora, Concepción y Sonoyta, que se planteó desde la instalación. El proyecto de creación del CICA buscaba contar con un instrumento moderno y eficaz para reunir información sobre el agua y apoyar a los usuarios de las cuencas y acuíferos del Alto Noroeste. Para ello se destinó un local en el edificio de la Unión Ganadera Regional de Sonora (Mendívil Corral, 2007). Este asunto se trató en las sesiones desde el año 2000 hasta 2004; se conformó una comisión y hubo avances menores, pero al final no se concretó. Otro asunto similar fue la creación de una página web del CCAN, que se trató en las sesiones del 16 de marzo del año 2000 y del 6 de julio de 2001, pero nunca se concretó y, cuando menos hasta 2017 el organismo carecía de ese recurso.

Por otra parte, al amparo del CCAN y de los recursos que aportan la CONAGUA y la CEA, se han realizado estudios de los acuíferos, el monitoreo de la calidad del agua en Puerto Peñasco, el manejo sostenible del agua en la Costa de Hermosillo y la adecuación de los derechos de agua (Salmón, 2006). Sin embargo, estas acciones se pueden atribuir más propiamente a la Comisión que al CCAN como tal.

Otro ejemplo del papel que juega el CCAN en la gestión del agua de sus cuencas es su poca participación en la respuesta y atención a la contingencia del derrame tóxico en el río Sonora, del 6 de agosto de 2014 (Gobierno de la República, 2015). Este evento es considerado el desastre ecológico más grave de Sonora (Greenpeace, 2014) pero el CCAN no jugó un papel relevante en la respuesta y acciones posteriores. Después del derrame, la reunión siguiente del CCAN se realizó hasta el 17 de diciembre de 2014 y ahí, entre otros, se asentaron estos acuerdos:

- El GSE se da por enterado del informe realizado por el ingeniero Julio López Hernández [de Conagua], sobre los principales hechos y acciones realizadas 
por la CONAGUA y otras dependencias, en atención de la contingencia presentada en el río Sonora por derrame de sustancias tóxicas, provenientes de la Mina Buenavista del Cobre en Cananea, Sonora.

- Se refrenda el acuerdo de la 51 reunión de GSE del CCAN, que se refiere a integrar el proyecto de restauración hidrológica para la cuenca del río Sonora dentro del programa de gestión del CCAN.

- Se buscará concretar una reunión entre el CCAN con los diputados que conforman la comisión para la contingencia del río Sonora y la comisión Sonora para la contingencia ambiental río Sonora, para que éstas rindan un informe sobre la planeación de la remediación total de la contingencia (CONAGUA-OCNO, 2017).

Como se puede ver, el CCAN no participó directamente en la atención a la contingencia, sino que solo fue una especie de caja de resonancia o ente validador de las acciones de la CONAGUA. Todos los presidentes municipales y usuarios de agua del río Sonora se organizaron y realizaron numerosas reuniones y gestiones, pero no lo hicieron a través del CCAN.

En un ejercicio de clasificación de los acuerdos registrados en las actas de sesión hasta 2015 se obtuvo que 30 de los 240 (12.5\%) se relacionan directamente con la gestión del agua, mientras que los $210(87.5 \%)$ restantes se refieren más bien a asuntos administrativos y de organización interna.

Entonces, la revisión de los acuerdos apunta a que las sesiones del CCAN se han dedicado a atender asuntos administrativos y muy poco a la gestión del agua de la cuenca; además, tampoco se ocupa de conflictos como la escasez de agua en Hermosillo, y del racionamiento o tandeo del servicio de agua potable o la contaminación del río Sonora.

Esta conclusión es similar a la planteada por Sánchez Meza (2008) respecto a la ineficacia y la falta de operatividad del consejo de cuenca del río Sonora, para atender los problemas graves abordados en las sesiones de trabajo del Grupo de Seguimiento y Evaluación, y a la expuesta por Parra y Salazar (2017) en relación con la capacidad limitada del CCAN para desenvolverse conforme a los principios de la GIRH.

\section{Reflexión final}

Con los componentes del concepto de capacidad institucional propuestos, se pueden revisar los elementos de la capacidad institucional del CCAN.

Primero, en cuanto a la autoridad legal, los consejos de cuenca no son autónomos sino órganos dependientes de la CONAGUA. Esto se aprecia en su composición formal ya que, al menos en su periodo inicial, el director general de la Comisión fungía como su presidente y el director del organismo de cuenca correspondiente como su secretario técnico.

Asimismo, las facultades otorgadas a los consejos de cuenca, definidas tanto en la LAN (art. 13 bis 3) (DOF, 2004) y en el reglamento de la LAN (DOF, 2014) (art. 13), no incluyen facultades o autoridad directa para la gestión, adminis- 
tración o planeación de la cuenca. Por los verbos que se utilizan (contribuir, concertar, conocer, promover), estas facultades son más bien de apoyo para la CONAGUA y no asignan responsabilidad ejecutiva sobre la cuenca. Por ello, los consejos de cuenca a lo sumo son órganos consultivos, orientados a convalidar o a ser caja de resonancia de la agenda de la Comisión. Entonces, resulta engañoso equiparar a los consejos de cuenca de México con los de otros países, donde son responsables directos de la planeación y gestión del agua de sus cuencas (Cap-Net UNDP, 2008; Huitema y Meijerink, 2014).

Tabla 5. Resumen de hallazgos

sobre la capacidad institucional del CCAN

\begin{tabular}{|c|c|c|}
\hline Dimensiones & Variables & Resultados observados \\
\hline \multirow[b]{2}{*}{$\begin{array}{l}\text { Autoridad } \\
\text { legal }\end{array}$} & $\begin{array}{c}\text { Facultades } \\
\text { legales para } \\
\text { gestionar el agua }\end{array}$ & $\begin{array}{c}\text { No cuenta con facultades legales para gestionar } \\
\text { el agua, ya que la LAN ni su reglamento le } \\
\text { asignan autoridad para la gestión del agua }\end{array}$ \\
\hline & $\begin{array}{l}\text { Toma de } \\
\text { decisiones para } \\
\text { gestión del agua }\end{array}$ & $\begin{array}{l}\text { En la agenda de las reuniones prevalecen } \\
\text { asuntos no relacionados con la gestión del } \\
\text { agua de la cuenca, sino con información } \\
\text { y la aprobación de otros más amplios } \\
\text { propuestos por la CONAGUA }\end{array}$ \\
\hline \multirow[b]{2}{*}{$\begin{array}{l}\text { Integración } \\
\text { y dirección } \\
\text { del consejo }\end{array}$} & $\begin{array}{l}\text { Integración y } \\
\text { estructura }\end{array}$ & $\begin{array}{c}\text { El liderazgo del CCAN reside en la CONAGUA } \\
\text { y su composición es gubernamental. } \\
\text { Son funcionarios de la Comisión, } \\
\text { quienes presiden y organizan las sesiones }\end{array}$ \\
\hline & $\begin{array}{l}\text { Nombramiento } \\
\text { director o } \\
\text { presidente } \\
\text { del CCAN }\end{array}$ & $\begin{array}{c}\text { Por disposición de la LAN, el presidente primero } \\
\text { fue el director general de la CONAGUA. Después } \\
\text { de una reforma legal, la presidencia recayó en } \\
\text { el gerente del organismo. A partir de } 2015 \text { se } \\
\text { comenzó a elegir a un usuario de agua como } \\
\text { presidente, pero el secretario técnico continúa } \\
\text { siendo un funcionario de la CONAGUA. } \\
\text { Ha habido un avance relativo } \\
\text { en la participación de los usuarios }\end{array}$ \\
\hline \multirow{2}{*}{ Participación } & $\begin{array}{l}\text { Frecuencia } \\
\text { de reuniones }\end{array}$ & $\begin{array}{c}\text { En el periodo estudiado hubo, en promedio, } \\
3.84 \text { reuniones por año; } \\
\text { es una organización relativamente activa }\end{array}$ \\
\hline & $\begin{array}{l}\text { Participación } \\
\text { de miembros }\end{array}$ & $\begin{array}{l}\text { Asistieron, en promedio, } 20.7 \text { personas } \\
\text { por sesión, la mayoría representantes } \\
\text { de dependencias de gobierno }\end{array}$ \\
\hline \multirow[t]{2}{*}{$\begin{array}{l}\text { Capacidad } \\
\text { financiera }\end{array}$} & Presupuesto & $\begin{array}{c}\text { En } 2008 \text { recibió } \$ 1300000 \text { y aproximadamente } \\
\$ 1200000 \text { anuales en los años siguientes. } \\
\text { En } 2017 \text { fue de } \$ 670000 . \\
\text { Depende del apoyo financiero de la CONAGUA, } \\
\text { y no tiene otros ingresos }\end{array}$ \\
\hline & Ingresos propios & $\begin{array}{c}\text { No hay cuotas de miembros, se sostiene con } \\
\text { aportaciones de la CONAGUA y la CEA }\end{array}$ \\
\hline
\end{tabular}

Fuente: elaboración propia, derivada de Cap-Net UNDP (2008). 
Otra observación es que, durante la mayor parte del periodo de estudio, la presidencia del CCAN recayó en funcionarios de la CONAGUA. Sin embargo, a partir de noviembre de 2015 se modificó esta regla y, en lo que se denominó el "perfeccionamiento" del consejo, se comenzó a elegir como presidente a un usuario de agua. Esto es un avance relativo, del control completo del consejo por parte de funcionarios de la Comisión, hacia una mayor participación de los usuarios de agua. Además, en su mayoría, su composición es gubernamental ya que formalmente tiende a prevalecer la representación de las dependencias federales y la coordinación con el gobierno estatal, más que los asuntos de los usuarios relacionados con los derechos de agua.

Las minutas y los acuerdos indican que los usuarios tienen deseos de participar, plantear propuestas y realizar acciones relacionadas con la gestión de la cuenca. Sin embargo, debido a que los consejos carecen de autoridad ejecutiva para decidir estos asuntos, se pierden incentivos para participar de manera más efectiva.

El CCAN depende de las aportaciones de CONAGUA y del gobierno de Sonora para su sostenimiento económico y, a lo largo de los años, éstas han tendido a disminuir. En este sentido, el organismo tiende a ser una dependencia gubernamental.

Por último, la revisión de las actas de las sesiones mostró que la participación de los usuarios es reducida, y que en las agendas y los acuerdos tomados prevalecen asuntos no relacionados directamente con la gestión del agua de las cuencas del alto noroeste, sino con la información y la aprobación de otros más amplios, propuestos por la CONAGUA.

La creación y existencia del Consejo de Cuenca del Alto Noroeste constituye un avance con respecto a la nula participación anterior de los usuarios y de los gobiernos locales en la gestión del agua, pero ésta todavía dista de lo que se puede considerar como efectiva en la planeación y gestión del agua de la cuenca. El vaso de la participación social en este aspecto se puede ver medio lleno, en el sentido de que representa un avance, pero no es suficiente; y medio vacío, porque muestra todas las deficiencias de las políticas verticales (de arriba hacia abajo), de la centralización de la CONAGUA, mucha rigidez y acartonamiento en las formas, y sus procedimientos todavía están alejados de despertar la energía, la innovación y el capital social que significa la participación social de abajo hacia arriba. Los consejos de cuenca en México en general, y el CCAN carecen de capacidad institucional para la gestión del agua de sus cuencas y, más bien, son órganos complementarios de la Comisión Nacional del Agua que está encargada efectivamente de la gestión de las cuencas, y mantiene el centralismo de la política hídrica en México.

\section{Agradecimientos}

Este trabajo de investigación fue posible gracias al apoyo brindado por el Consejo Nacional de Ciencia y Tecnología (Proyecto CB-2013/223780 “Vulnerabilidad hídrica y formulación de políticas adaptativas para la sustentabilidad urbana y 
la gestión por cuencas". Así como a la Lloyd's Register Foundation [CE-12-1051/ CE-12-0801] y al Instituto Inter-Americano para la Investigación del Cambio Global (IAI) [SGP-CRA \#005], el cual es apoyado por la National Science Foundation [GEO-1138881 y DEB-1010495]. Se reconoce y agradece también el trabajo de las asistentes de investigación Daniela Noreña, Melisa Aguirre Loreto, Alejandra Gallardo Urrea y Miryam Fernanda Ramírez Ruiz.

\section{Referencias}

Aboites Aguilar, L. (1998). El agua de la nación. Una historia política de México (1888-1946). México: Centro de Investigaciones y Estudios Superiores en Antropología Social.

Aguilar Villanueva, L. F. (2013). Gobierno y administración pública. México: Fondo de Cultura Económica y Consejo Nacional para la Cultura y las Artes.

Cap-Net UNDP. (2008). Performance and capacity in river basin organizations. Cross-case comparison of four RBOs. CAP-NET UNDP.

Comisión Estatal del Agua, C. d. (13 de mayo de 2013). Transparencia.esonora.gob.mx. Recuperado de http://transparencia.esonora.gob.mx/NR/rdonlyres/6315FB39-F2F2-48C3-8935-5CA85ED5AADA/115949/CCAN2013.pdf

Comisión Nacional del Agua. (1999). Acta constitutiva y de establecimiento del Consejo de Cuencas del Alto Noroeste. Hermosillo, Sonora.

Comisión Nacional del Agua-Comisión Estatal del Agua. (2008-2017). Convenios de Coordinación entre la Comisión Nacional del Agua y la Comisión Estatal del Agua 2008-2017. Gobierno del Estado de Sonora, Comisión Estatal del Agua. Hermosillo: Transparencia Sonora.

Comisión Nacional del Agua-Organismo de Cuenca del Noroeste. (2017). Minutas del Consejo de Cuenca del Alto Noroeste 1999-2017. Hermosillo, Sonora: Comisión Nacional del Agua- Organismo de Cuenca del Noroeste.

Diario Oficial de la Federación. (1 de diciembre de 1992). Ley de Aguas Nacionales. Secretaría de Agricultura y Recursos Hidráulicos.

Diario Oficial de la Federación. (12 de enero de 1994). Reglamento de la Ley de Aguas Nacionales. Secretaría de Agricultura y Recursos Hidráulicos.

Diario Oficial de la Federación. (10 de diciembre de 1997). Decreto que reforma el Reglamento de la Ley de Aguas Nacionales. Secretaría de Medio Ambiente, Recursos Naturales y Pesca.

Diario Oficial de la Federación. (29 de abril de 2004). Decreto por el que se reforma y adiciona y deroga diversas disposiciones de la Ley de Aguas Nacionales. Secretaría del Medio Ambiente y Recursos Naturales.

Diario Oficial de la Federación. (30 de noviembre de 2006). Reglamento Interior de la Comisión Nacional del Agua. Secretaría del Medio Ambiente y Recursos Naturales.

Diario Oficial de la Federación. (25 de agosto de 2014). Reglamento de la Ley de Aguas Nacionales. Secretaría del Medio Ambiente y Recursos Naturales.

Diccionario Esencial de la Real Academica Española. (1997). Madrid: ESPASA. 
Dunbier, R. (1968). The Sonoran Desert: Its geography, economy, and people. Tucson: The University of Arizona Press.

Gobierno de la República. (2015). Balance de las acciones del Gobierno de la República en el río Sonora. Secretaría del Medio Ambiente y Recursos Naturales, Comisión Federal para la Protección contra Riesgos Sanitarios. México: Fideicomiso Río Sonora.

Greenpeace México. (21 de agosto de 2014). Desastre en Sonora: la gran tragedia-la gran amenaza. Recuperado de http://www.greenpeace.org/mexico/ es/Noticias/2014/Agosto/Desastre-en-Sonora-La-gran-tragedia---La-granamenaza/

Hooper, B. P. (2006). Performance Indicators of River Basin Organizations. Carbondale: US Army Corps of Engineers \& IWR.

Hooper, B. (2010). River basin organization performance indicators: applications to the Delaware River Basin Commission. Water Policy, 12, 1-24.

Huitema, D., y Meijerink, S. (2014). The politics of river basin organisations: Coalitions, institutional design choices and consequences. Cheltenham: Edward Elgar.

Mendívil Corral, A. L. (2007). Participación social en la gestión de un Centro de Información y Consulta sobre el Agua del Consejo de Cuenca del Alto Noroeste (Tesina). El Colegio de Sonora, Hermosillo.

Mercadante, G. (21 de noviembre de 2017). Gerente Operativo del CCAN. Entrevistada por Nicolás Pineda Pablos.

North, D. (1990). Institutions, institutional change and economic performance. Cambridge: Cambridge University Press.

Parra Armenta, E., y Salazar Adams, A. (2017). La gestión integral del agua en dos consejos de cuenca del noroeste de México. Entreciencias, 5(15), 79-94. doi: 10.22201/enesl.20078064e.2017.15.62580

Rodríguez, V. (1997). Decentralization in Mexico. From reforma municipal to solidaridad to nuevo federalismo. Boulder: Westview Press.

Salmón, R. (2006). Avances, logros, retos y oportunidades de los consejos de cuenca 2001-2006. Hermosillo: Comisión Nacional del Agua.

Sánchez Meza, J. J. (2008). El mito de la gestión descentralizada en México. México: Instituto de Investigaciones Jurídicas, Universidad Nacional Autónoma de México.

VanHofwegen, P. J., y Jaspers, F. G. (2000). Marco analítico para el manejo integrado de los recursos hídricos. Lineamientos para evaluar el marco institucional. Washington: Banco Interamericano de Desarrollo. 